\title{
Electrostatically driven complex coacervation and amyloid aggregation of tau are independent processes with overlapping conditions
}

\author{
Yanxian Lin ${ }^{1}$, Yann Fichou ${ }^{2}$, Zhikai Zeng ${ }^{2}$, Nicole Y. Hu³ ${ }^{3}$ Songi Han²,3 \\ ${ }^{1}$ Biomolecular Science and Engineering, University of California, Santa Barbara, CA 93106 \\ ${ }^{2}$ Department of Chemistry and Biochemistry, University of California, Santa Barbara, CA 93106 \\ ${ }^{3}$ Department of Chemical Engineering, University of California, Santa Barbara, CA 93106
}

\section{Figure S1}

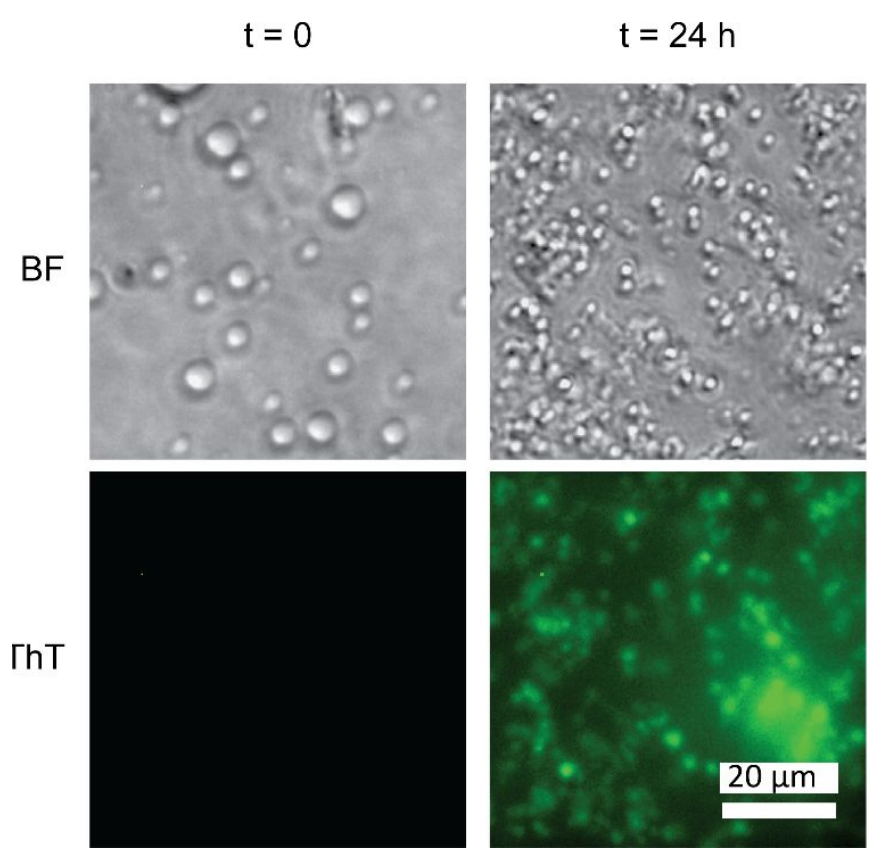

Microscope images of tau-heparin LLPS and amyloid aggregation at room temperature. $100 \mu \mathrm{M}$ tauS was incubated with $170 \mu \mathrm{g} / \mathrm{mL}$ heparin and $10 \mu \mathrm{M}$ ThT at room temperature for $24 \mathrm{~h}$. Bright field (BF) images and fluorescence of ThT $\left(\lambda_{\text {emission }}=485\right.$ $\mathrm{nm})$ were taken immediately after mixing $(\mathrm{t}=0 \mathrm{~h})$, and 24 hours after incubation $(\mathrm{t}=24 \mathrm{~h})$. Scale bar is $20 \mu \mathrm{m}$ long. Results in this figure are representative. 
Figure S2
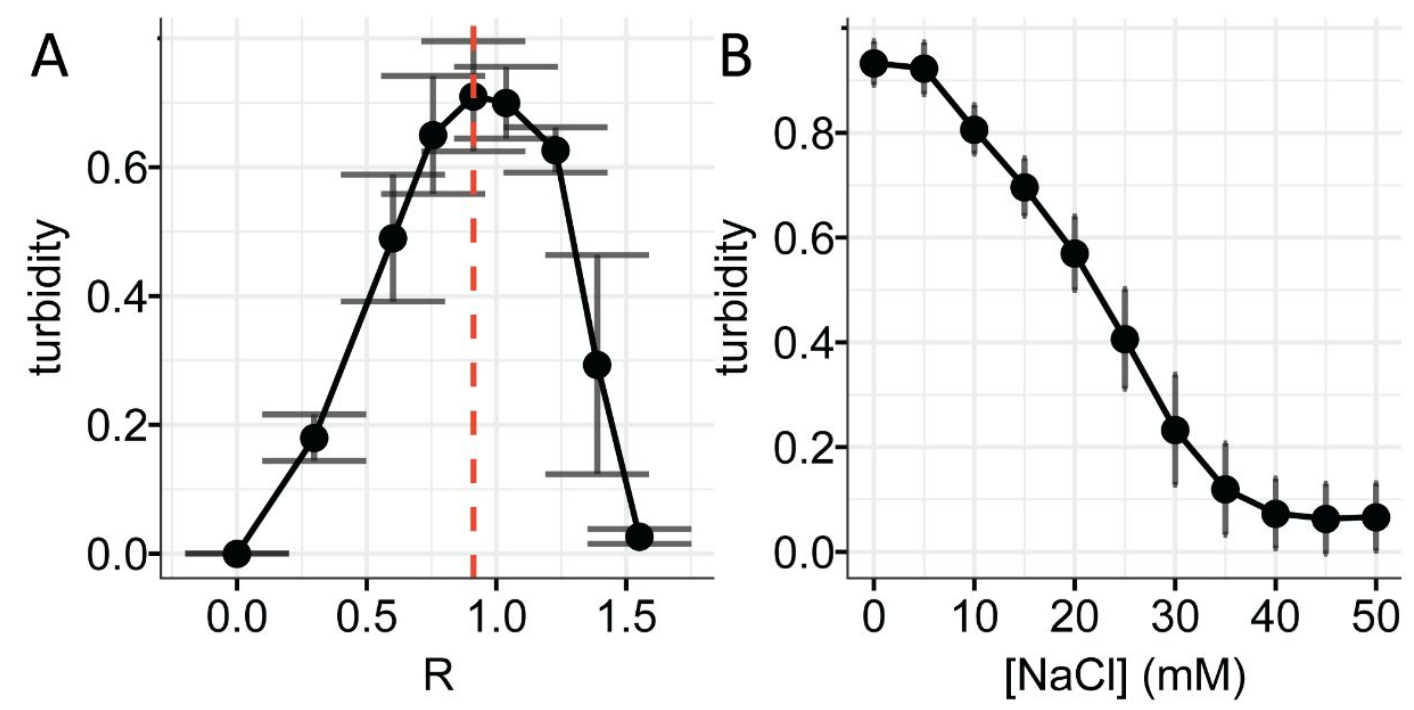

Turbidity dependence of tau-heparin LLPS at varying charge ratio and ionic strength.

A. Turbidity of tauS-heparin mixture at varying charge ratio (R). [tau $]=20 \mu \mathrm{M},[\mathrm{NaCl}]=$ $0 . \mathrm{R}=1$ corresponds to $1.7 \mu \mathrm{g} / \mathrm{mL}$ heparin per $1 \mu \mathrm{M}$ tau. Dashed line shows the position of the peak reading. B. turbidity of tau-heparin at varying [ $\mathrm{NaCl}]$ from 0 to $50 \mathrm{mM}$. [tau] $=20 \mu \mathrm{M}, \mathrm{R}=1$. Error bars are standard deviation of 3 independent technical replicates. 
Figure S3

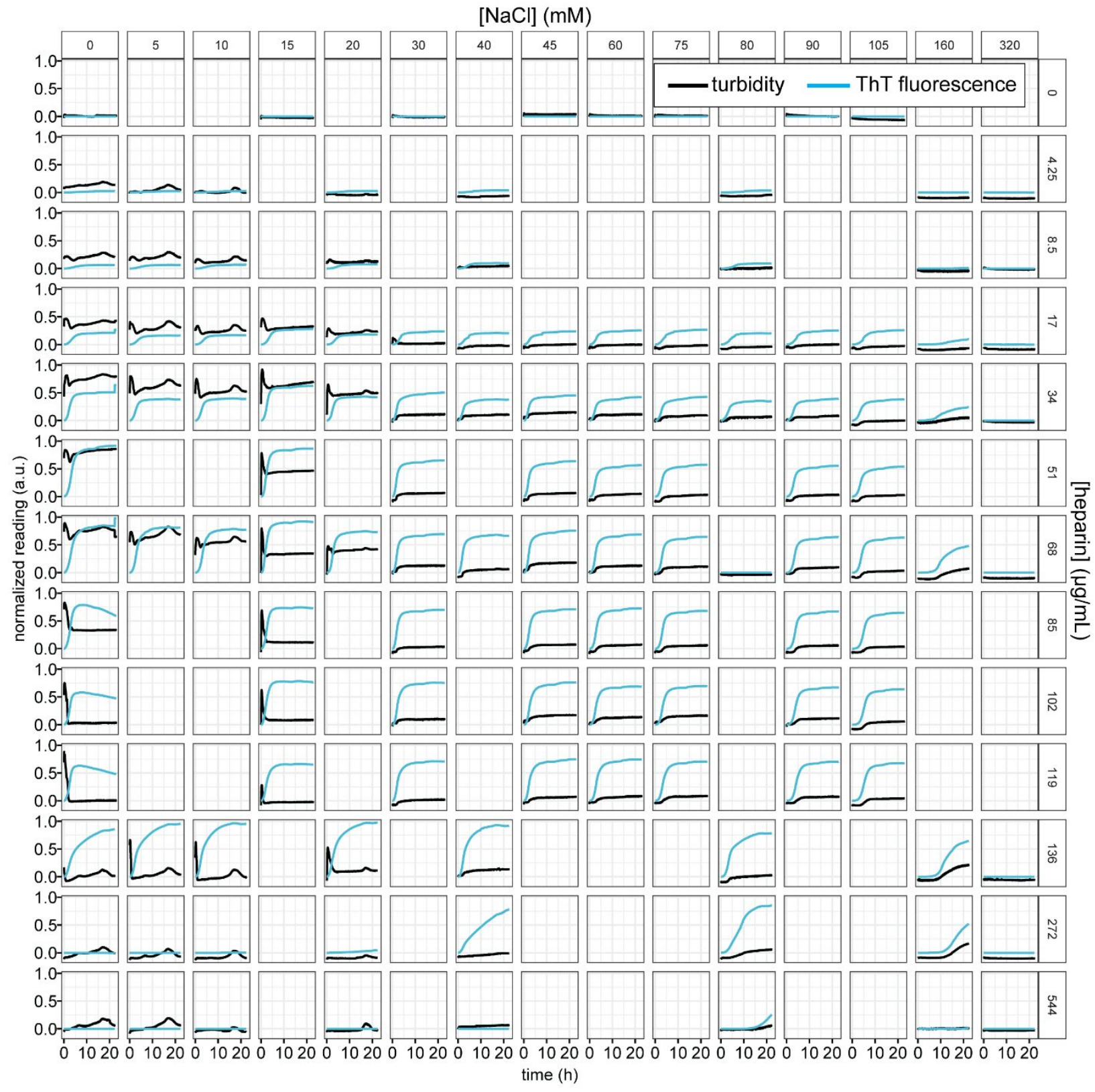

ThT fluorescence and turbidity data of Figure 2. $20 \mu \mathrm{M}$ of tauSS was incubated with heparin at varying [heparin] and [ $\mathrm{NaCl}]$. Turbidity (black) and ThT fluorescence (blue) of each sample were monitored and scaled to $0 \sim 1$. Different rows correspond to varying [heparin] from 0 to $544 \mu \mathrm{g} / \mathrm{mL}$. Different columns correspond to varying [ $\mathrm{NaCl}]$ from 0 to $320 \mathrm{mM}$. Results in this figure come from three different batches of protein. 
Figure S4
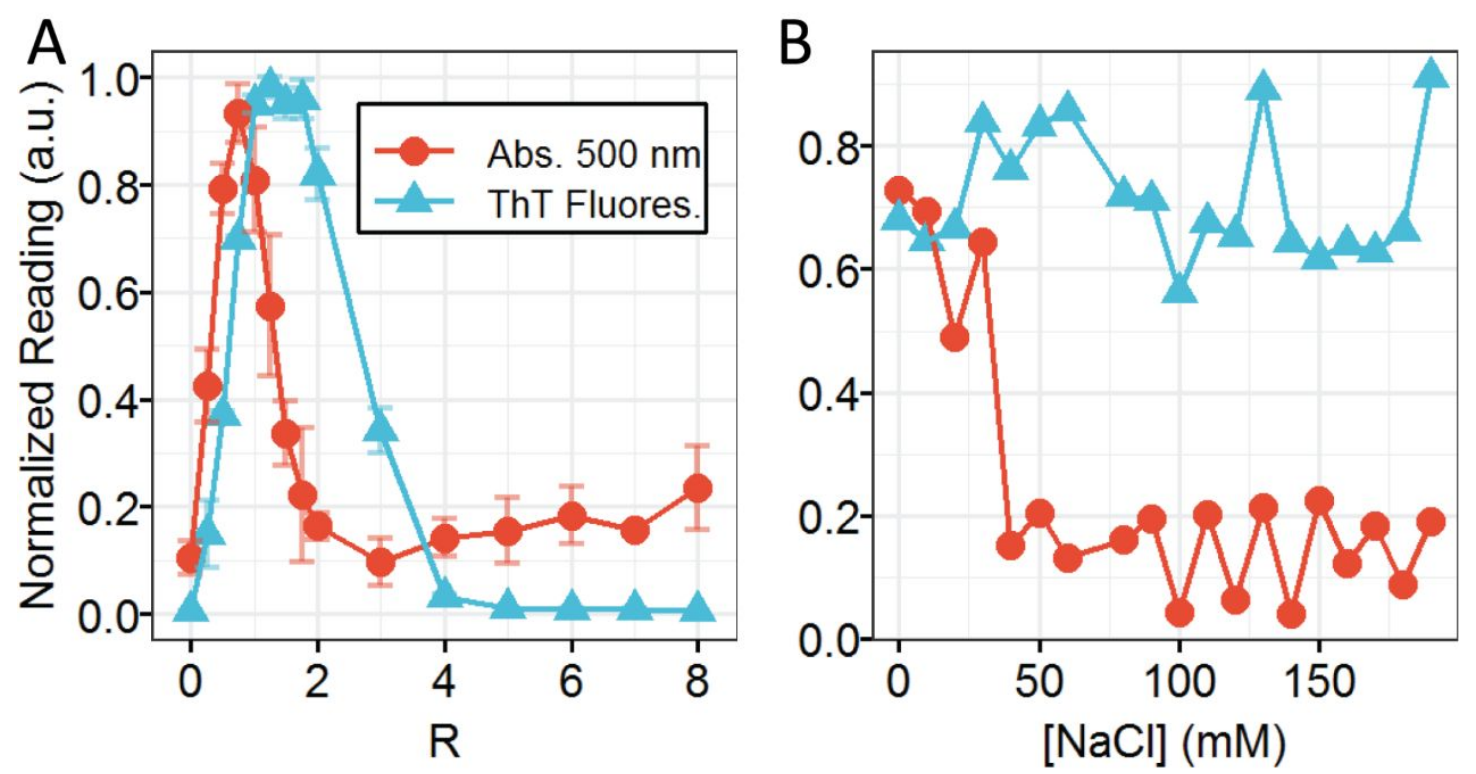

Turbidity and ThT fluorescence of tauS-heparin. A. Normalized Turbidity and ThT fluorescence of tau187C291S-heparin aggregation at different charge ratio, R. [tau] $=20$ $\mu \mathrm{M},[\mathrm{NaCl}]=0 \mathrm{mM} . \mathrm{R}=1$ corresponds to $[$ heparin $]=34 \mu \mathrm{g} / \mathrm{mL}$. Error bars show standard deviation of 3 technical replicates. B. Normalized turbidity and ThT fluorescence of tauheparin aggregation at different $[\mathrm{NaCl}]$. $[\mathrm{tau}]=20 \mu \mathrm{M}$. Charge ratio $\mathrm{R}=1$. Result in $\mathrm{B}$ is representative. 
Figure S5
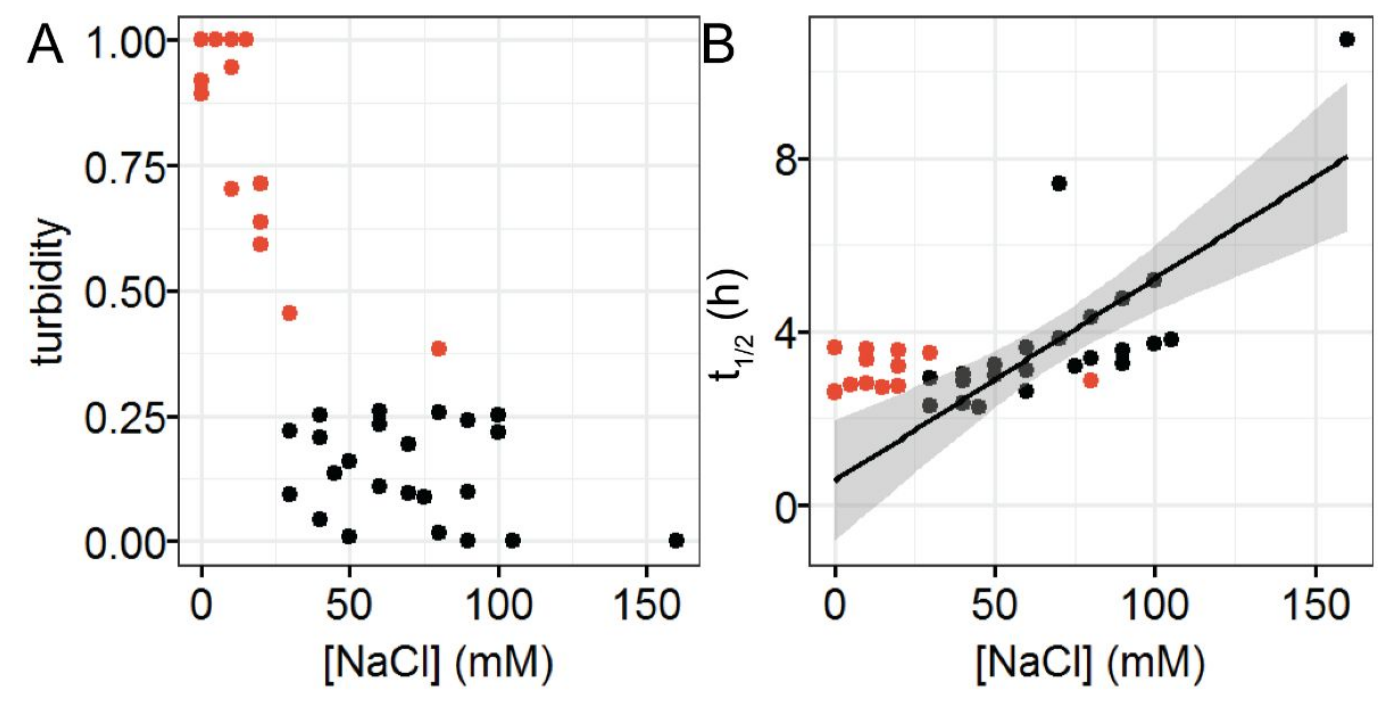

Effects of LLPS on tau-heparin aggregation half time. A. Normalized turbidity reading of tauSS-heparin mixture at varying $[\mathrm{NaCl}]$. Data points are independent replicates from three different batches of protein. B. $t_{1 / 2}$ of tauSS-heparin aggregation at different $[\mathrm{NaCl}]$. $20 \mu \mathrm{M}$ tauSS with $34 \mu \mathrm{g} / \mathrm{mL}$ heparin were used. In both $\mathrm{A}$ and $\mathrm{B}$, data points with normalized turbidity above 0.3 are colored in red. 
Figure S6
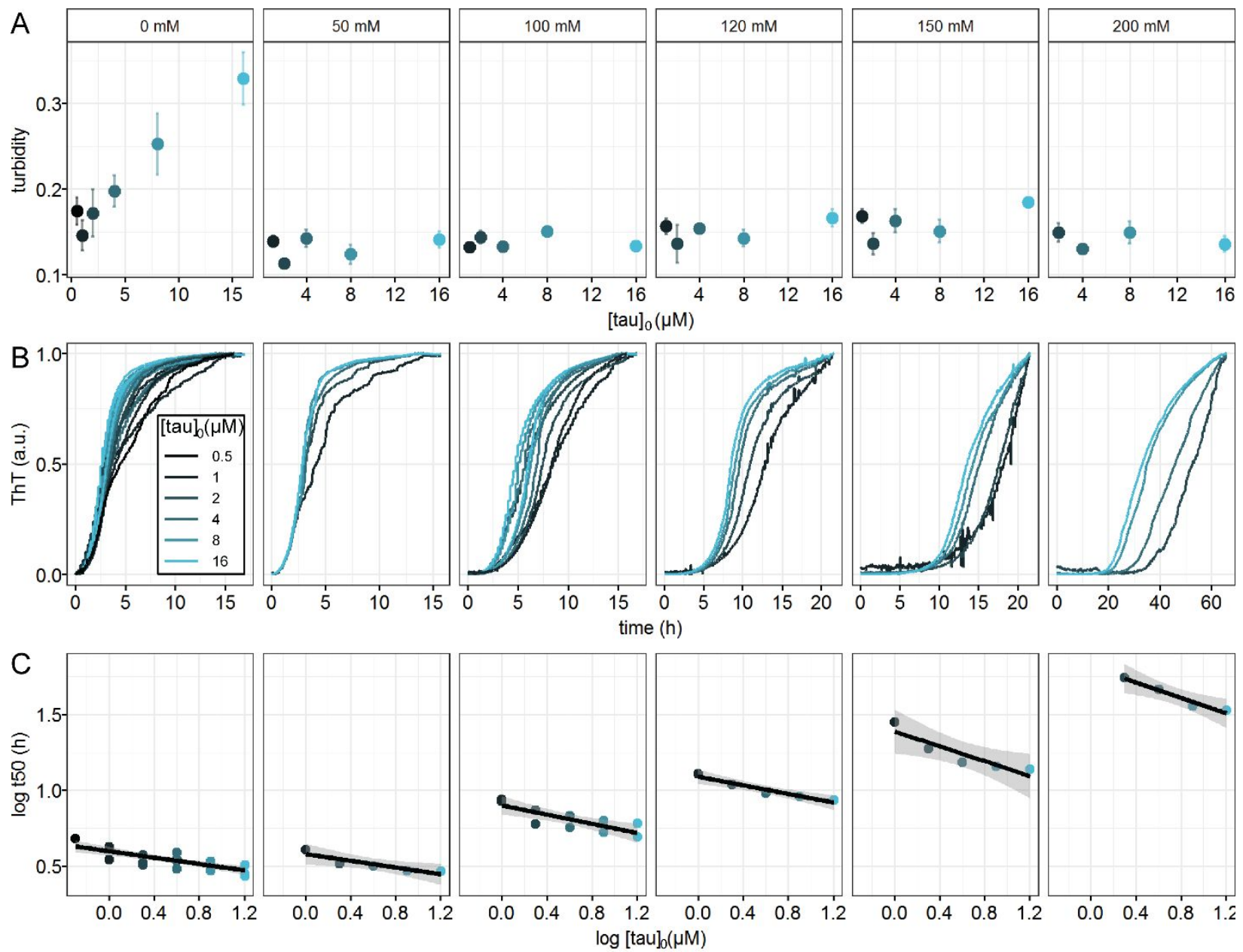

Effects of LLPS on tau-heparin aggregation monomer dependence. A. Turbidity of tauSS-heparin mixture at varying initial tau concentration, $[\operatorname{tau}]_{0}$. Ratio of heparin was fixed at $1.7 \mu \mathrm{g} / \mathrm{mL}$ heparin per $1 \mu \mathrm{M}$ tau. B. Normalized ThT fluorescence of tauSS-heparin mixture at varying $[\mathrm{tau}]_{0}$ and $[\mathrm{NaCl}]$. Data were fit with sigmoid function to extract $\mathrm{t}_{1 / 2}$ (see Materials and Methods). C. $\log -\log$ plot of $\mathrm{t}_{1 / 2} \mathrm{vs}[\mathrm{tau}]_{0}$. Solid lines are linear regression of the data points. Shade areas show confidence interval of the regression. Slopes of the fit, or $\gamma$, are $-0.11 \pm 0.02,-0.11 \pm 0.03,-0.15 \pm 0.04,-0.14 \pm 0.02,-0.24 \pm 0.06$, $-0.26 \pm 0.04$, for each panel from left to right respectively. $\mathrm{R}^{2}$ of the regression are 0.64 , $0.82,0.68,0.94,0.84,0.95$, for each panel from left to right respectively. Results in this figure come from biological replicates using three different batches of protein. 
Figure S7
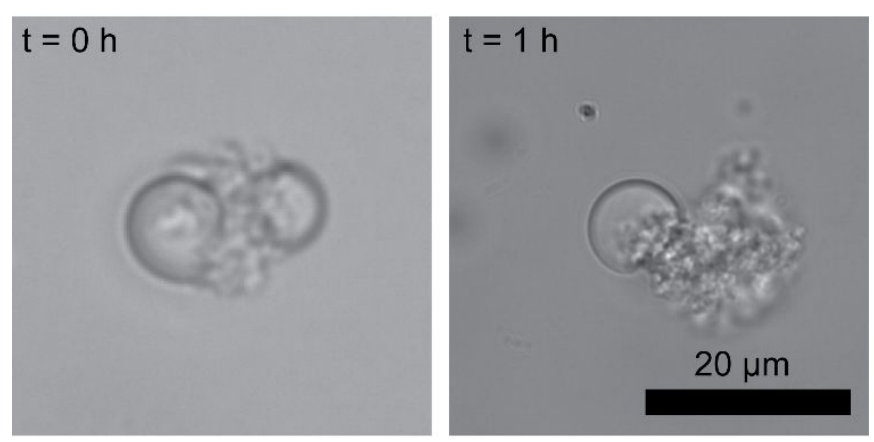

Direct contacts of tau-RNA droplets with tau aggregates. $20 \mu \mathrm{M}$ tauSSP301L was mixed with $60 \mu \mathrm{g} / \mathrm{mL}$ poly(U) RNA in the presence of $1 \mu \mathrm{M}$ seed (prepared as below). Samples were loaded onto a microscope slide with glass cover slide and incubated at room temperature for 1 hour before images were taken. Seed was prepared by incubating $20 \mu \mathrm{M}$ tauSSP301L with $5 \mu \mathrm{M}$ heparin overnight at $37^{\circ} \mathrm{C}$. Results in this figure are representative. 
Figure S8

A

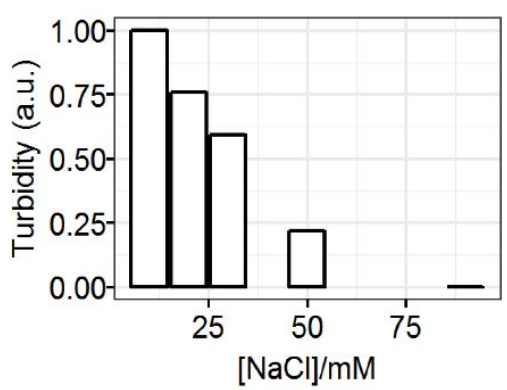

C

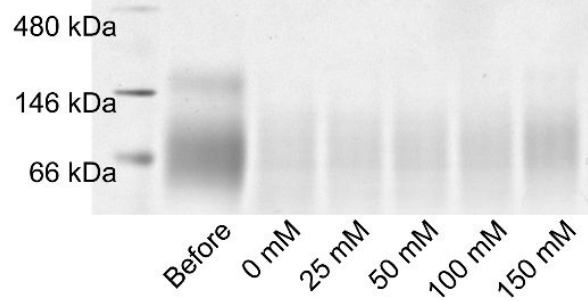

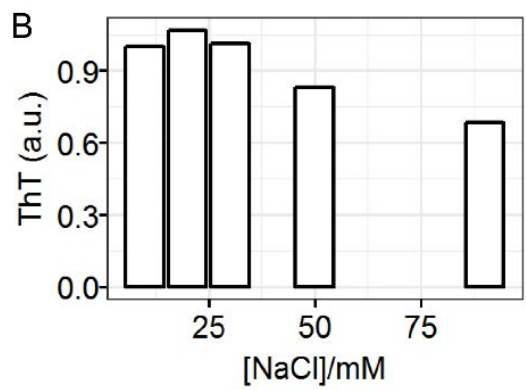

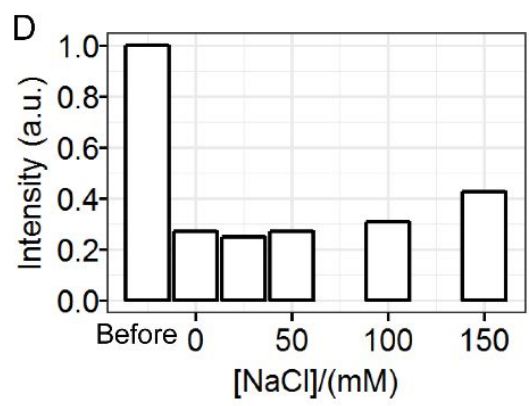

ThT fluorescence of tau-heparin LLPS correlates with amount of amyloid aggregates.

$100 \mu \mathrm{M}$ of tauSS was mixed with $170 \mu \mathrm{g} / \mathrm{mL}$ heparin in the presence of varying $\mathrm{NaCl}$. A. Mean turbidity of samples at the initial 1 hour. B. Mean ThT fluorescence of samples after incubation. C. Blue Native PAGE of samples after incubation. D. Integrated band intensity of C. Results in this figure are representative. 


\section{Figure S9}
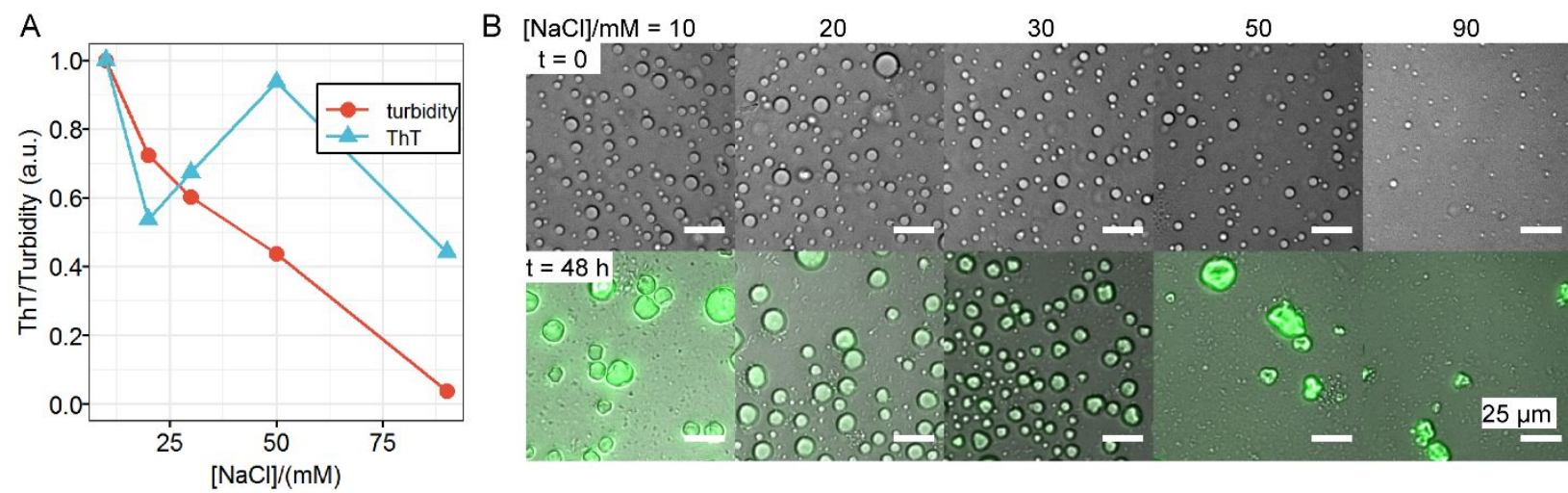

LLPS-CC and amyloid aggregation of full length 2N4R-heparin. $10 \mu \mathrm{M}$ of 2N4RC291SC322S was mixed with $17 \mu \mathrm{g} / \mathrm{mL}$ heparin for an estimated charge ratio of $\mathrm{R}$

$=1$. Samples were incubated at room temperature for 48 hours in the presence of varying additional $\mathrm{NaCl}$. A. Initial (mean of $\mathrm{t}=0-1 \mathrm{~h}$ ) reading of turbidity normalized by samples at $\mathrm{t}=0 \mathrm{mM}$ and $90 \mathrm{mM}$, compared with final (mean of $\mathrm{t}=48 \mathrm{~h}$ ) readings of ThT scaled by reading at $\mathrm{t}=0 \mathrm{mM}$. B. Microscope images of bright field superimposed with ThT fluorescence (green) of samples at initial and final stages of incubation. Results in this figure are representative. 


\section{Figure S10}

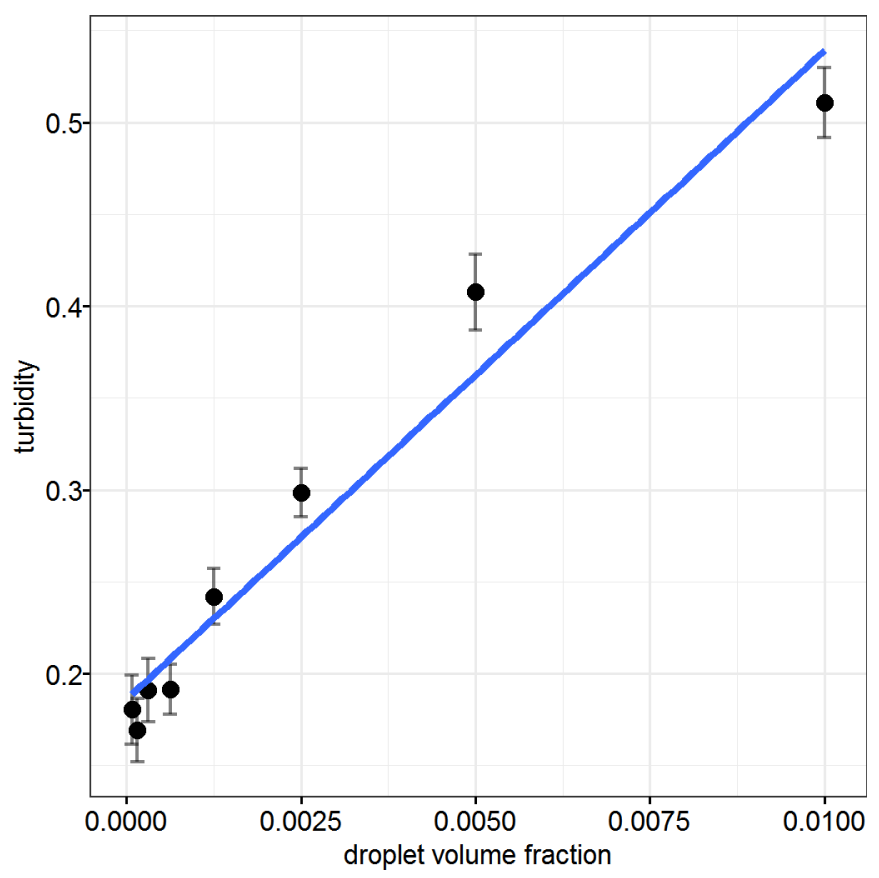

Turbidity vs droplet volume fraction of LLPS-CC formed by hyaluronic acid and

poly-L-lysine. We got the materials Hyaluronic acid (HA) and poly-L-lysine (PLL), as well as the idea of using such system from Dongsoo Hwang. HA and PLL stocks were prepared in sodium acetate buffer $(\mathrm{pH} \mathrm{5.0)}$ and mixed by 5:1 HA:PLL volume ratio to prepare LLPS-CC. Phase separated mixture was centrifuged at $1000 \mathrm{rcf}$ for $5 \mathrm{~min}$, resulting in dense phase separated from dilute phase. Dense and dilute phases were then collected and mixed at various ratio to prepare samples of given droplet volume fraction. Turbidity readings were acquired, plot against volume fraction (black points), and fit with linear regression (blue line). Error bars show standard deviation of 5 technical repeats. 


\section{Figure S11}
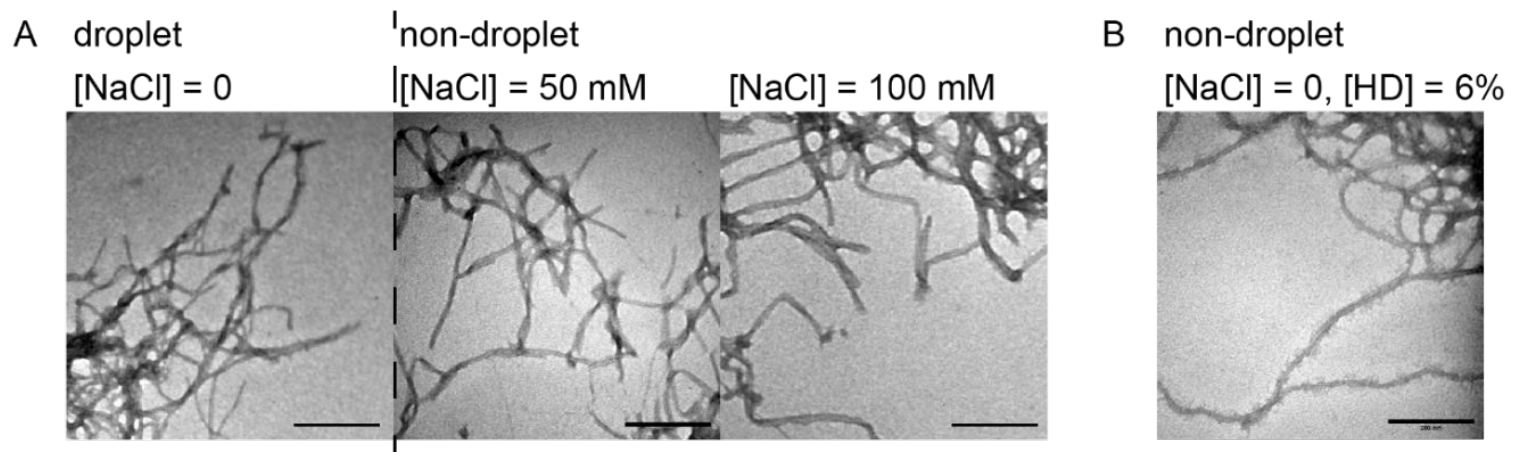

Representative TEM images of tau-heparin samples after incubation. $20 \mu \mathrm{M}$ tauSS was mixed with $34 \mu \mathrm{g} / \mathrm{mL}$ heparin in low ionic strength condition to prepare droplet sample (LLPS-CC). Either additional $50 \mathrm{mM}$ and $100 \mathrm{mM} \mathrm{NaCl}(\mathbf{A})$, or additional $6 \mathrm{wt} \%$ of hexanediol (HD) (B) was added to eliminate droplets. Of all samples in this figure, mixture was incubated overnight until ThT fluorescence reached plateau. Samples were than used to prepare TEM grids (fcf-cu-200, EMS), and images were acquired with a JEOL JEM1230 (JEOL USA, Inc) electron microscopy. Length of all scale bars is $200 \mathrm{~nm}$. 